\title{
Inverse Multi-Objective Shortest Path Problem Under the Bottleneck Type Weighted Hamming Distance
}

\author{
Mobarakeh Karimi ${ }^{1}$, Massoud Aman ${ }^{2 \star}$ and Ardeshir Dolati ${ }^{3}$ \\ 1 Department of Mathematics, University of Birjand, Birjand, Iran \\ mobarake.karimi@birjand.ac.ir \\ 2 Department of Mathematics, University of Birjand, Birjand, Iran \\ mamann@birjand.ac.ir \\ 3 Department of Computer Science, Shahed University, Tehran, Iran \\ dolati@shahed.ac.ir
}

\begin{abstract}
Given a network $G=(N, A, C)$ and a directed path $P^{0}$ from the source node $s$ to the sink node $t$, an inverse multi-objective shortest path problem is to modify the cost matrix $C$ so that $P^{0}$ becomes an efficient path and the modification is minimized. In this paper, the modification is measured by the bottleneck type weighted Hamming distance and is proposed an algorithm to solve the inverse problem. Our proposed algorithm can be applied for some other inverse multiobjective problem. As an example, we will mention how the algorithm is used to solve the inverse multi-objective minimum spanning tree problem under the bottleneck type weighted Hamming distance.
\end{abstract}

Keywords: Multi-objective optimization, Shortest path problem, Inverse problem, Hamming distance

\section{Introduction}

The inverse shortest path problem (ISPP) is one of the most typical problems of the inverse optimization, which is to make a predetermined solution becomes the optimal solution after modifications. This problem has attracted many attentions recently due to its broad applications in practice such as the traffic modeling and the seismic tomography (see, e.g., [5] and [10]). For example, assume that in a road network, we would like to modify the costs of the crossing such that a special path between two given nodes becomes optimum in order that, for some reason, the users select this path. To do this, we need to solve an ISPP.

In 1992, Burton and Toint [2] first formulated the ISPP using the $l_{2}$ norm to measure the modification. Zhang et. al [13] showed that the ISPP is equivalent to solving a minimum weight circulation problem when the modifications are measured by the $l_{1}$ norm. In [12], a column generation scheme is developed to solve the ISPP under the $l_{1}$ norm. Ahuja and Orlin [1] showed that the

\footnotetext{
* corresponding author.
} 
ISPP under the $l_{1}$ norm can be solved by solving a new shortest path problem. For the $l_{\infty}$ norm, they showed that the problem reduces to a minimum mean cycle problem. In [11], it is shown that all feasible solutions of the ISPP form a polyhedral cone and the relationship between this problem and the minimum cut problem is discussed. Duin and Volgenant [3] proposed an efficient algorithm based on the binary search technique to solve the ISPP under the bottleneck type Hamming distance (BWHD). In [9], Tayyebi and Aman extended their method to solve the inverse minimum cost flow problem and the inverse linear programming problem.

As with most real-world optimization problems, there is usually more than one objective that has to be taken into account, thus leading to multi-objective optimization problems (MOP) and inverse multi-objective optimization problems (IMOP). IMOP consists of finding a minimal adjustment of the objective functions coefficients such that a given feasible solution becomes efficient. Ronald et. al. [7] proposed an algorithm to solve the inverse multi-objective combinatorial optimization problems under the $l_{\infty}$ norm.

In this paper, we propose an algorithm to solve the inverse multi-objective shortest path problem under the BWHD. Our proposed algorithm can be used for solving the inverse of the multi-objective version of some problems under the BWHD. As an example, we apply the algorithm for the inverse multi-objective minimum spanning tree problem under the BWHD.

\section{Preliminaries}

The notations and definitions used in this paper are given in this section. Let $x, y \in \mathbb{R}^{q}$ be two vectors. $x \leq y$ iff $x_{k} \leq y_{k}$ for every $k \in\{1, \ldots, q\}$ and $x \neq y$. Let $G=(N, A, C)$ be a directed network consisting of a set of nodes $N=\{1,2, \ldots, n\}$, a set of $\operatorname{arcs} A \subseteq N \times N$ with $|A|=m$ and a cost matrix $C \in \mathbb{R}^{m \times q}$. In the matrix $C$, we denote the row corresponding to the arc $a \in A$ by the vector $C(a)$. This vector is called the cost of the arc $a$. The element $k$ of $C(a)$ is denoted by $C^{k}(a)$. For each $i_{1}, i_{r} \in N$ a directed path from $i_{1}$ to $i_{r}$ in $G$ is a sequence of nodes and $\operatorname{arcs} i_{1}-a_{1}-i_{2}-a_{2}-\ldots-i_{r-1}-a_{r-1}-i_{r}$ satisfying the properties that for all $1 \leq k \leq r-1,\left(i_{k}, i_{k+1}\right) \in A$ and for all $k, l \in\{1, \ldots, r\}, i_{k} \neq i_{l}$ if $k \neq l$. For each path $P$ in $G$, the cost of $P$ is defined as $C(P)=\sum_{a \in P} C(a)$. A path $P$ from $i$ to $j$ is called an efficient path if there is no other path $P^{\prime}$ from $i$ to $j$ such that $C\left(P^{\prime}\right) \leq C(P)$. Let $s, t \in N$ be two given nodes called the source and sink node, respectively. The multi-objective shortest path problem (MSPP) is to find all efficient directed paths from $s$ to $t$.

Theorem 1. [8] The bicriterion shortest path problem is NP-complete.

In [4] the multi-objective label setting algorithm is presented in the case that the cost of the arcs is nonnegative. Also the multi-objective label correcting algorithm is presented in the other case.

For a given path $P^{0}$ from $s$ to $t$ in $G$, The inverse multi-objective shortest path problem (IMSPP) is to find a matrix $D \in \mathbb{R}^{m \times q}$ such that 
(a) $P^{0}$ is an efficient path in the network $G=(N, A . D)$;

(b) For each $a \in A$ and $k \in\{1, \ldots, q\},-L^{k}(a) \leq D^{k}(a)-C^{k}(a) \leq U^{k}(a)$, where $L^{k}(a), U^{k}(a) \geq 0$ are given bounds for modifying cost $C^{k}(a)$;

(c) The distance between $C$ and $D$ is minimized.

The distance between $C$ and $D$ can be measured by various matrix norms. Also these matrices can be converted to two vectors, by a vectorization method, and a vector norm is used. Let each arc $a$ has an associated penalty $w(a) \in \mathbb{R}_{\geq}^{q}$. In this paper, we use the BWHD defined as follows:

$$
H_{w}(C, D)=\max _{a \in A, k \in\{1, \ldots, q\}} w^{k}(a) \cdot H\left(C^{k}(a), D^{k}(a)\right),
$$

where $H\left(C^{k}(a), D^{k}(a)\right)$ is the Hamming distance, i.e.

$$
H\left(C^{k}(a), D^{k}(a)\right)=\left\{\begin{array}{lll}
1 & \text { if } \quad C^{k}(a) \neq D^{k}(a), \\
0 & \text { if } \quad C^{k}(a)=D^{k}(a) .
\end{array}\right.
$$

\section{The IMSPP under the BWHD}

In this section, the IMSPP under the BWHD is considered and an algorithm is proposed to solve it. Let $G=(N, A, C)$ be a network with a source node $s$ and a sink node $t$. Assume that $P^{0}$ is a given directed path from $s$ to $t$ in $\mathrm{G}$. We can write the IMSPP under the BWHD as follows:

$$
\begin{array}{cl}
\min & H_{w}(C, D), \\
\text { s.t. } & P^{0} \text { is an efficient path from } s \text { to } t \text { in } G=(N, A, D), \\
& -L^{k}(a) \leq D^{k}(a)-C^{k}(a) \leq U^{k}(a), \quad \forall a \in A, \quad \forall k \in\{1, \ldots, q\} \\
& D \in \mathbb{R}^{m \times q},
\end{array}
$$

where $w: A \rightarrow \mathbb{R}^{q}$ is the arc penalties function and for each $a \in A$ and $k \in$ $\{1, \cdots, q\}, L^{k}(a)$ and $U^{k}(a)$ are the bounds for modifying cost $C^{k}(a)$. Assume that $w_{1} \leq w_{2} \leq \ldots \leq w_{q m}$ denote the sorted list of the arc penalties. For each $k \in\{1, \ldots, q\}$ and $r \in\{1,2, \ldots, q m\}$, we define $A_{r}^{k}=\left\{a \in A: w^{k}(a) \leq w_{r}\right\}$ and the matrix $D_{r}$ with the following elements is defined:

$$
D_{r}^{k}(a)= \begin{cases}C^{k}(a) & \text { if } \quad a \in A \backslash A_{r}^{k}, \\ C^{k}(a)+U^{k}(a) & \text { if } \quad a \in A_{r}^{k} \backslash P^{0}, \\ C^{k}(a)-L^{k}(a) & \text { if } \quad a \in A_{r}^{k} \cap P^{0} .\end{cases}
$$

The following theorem provides a helpful result for presenting our algorithm.

Theorem 2. If $D$ is a feasible solution to the problem (3) with the objective value $w_{r}$, then $D_{r}$ defined in (4) is also feasible whose objective value is less than or equal to $w_{r}$.

Proof. It is easily seen that $D_{r}$ satisfies the bound constraints and its objective value is not greater than $w_{r}$. On the contrary, suppose that $P^{0}$ is not efficient 
in $G=\left(N, A, D_{r}\right)$. This means that there exists a path $P$ from $s$ to $t$ such that $D_{r}(P) \leq D_{r}\left(P^{0}\right)$. We prove that $D(P) \leq D\left(P^{0}\right)$. Hence $P^{0}$ is dominated by $P$ in $G=(N, A, D)$ which contradicts the feasibility of $D$ for (3).

The inequality $D_{r}(P)-D_{r}\left(P^{0}\right) \leq 0$ implies that

$$
\begin{aligned}
D(P)-D\left(P^{0}\right) & \leq D_{r}\left(P^{0}\right)-D_{r}(P)+D(P)-D\left(P^{0}\right) \\
& =\sum_{a \in P^{0}}\left(D_{r}(a)-D(a)\right)+\sum_{a \in P}\left(D(a)-D_{r}(a)\right) \\
& =\sum_{a \in\left(P^{0} \backslash P\right)}\left(D_{r}(a)-D(a)\right)+\sum_{a \in\left(P \backslash P^{0}\right)}\left(D(a)-D_{r}(a)\right) .
\end{aligned}
$$

By the definition of $D_{r}$ and feasibility of $D$ for (3), all the phrases of the right hand side of (5) are nonpositive. Therefore $D(P)-D\left(P^{0}\right) \leq 0$, which completes the proof.

The following theorem is concluded immediately from Theorem 2 .

Corollary 1. If the optimal objective value of (3) is $w_{r}$, then $D_{r}$ defined in (4) is an optimal solution to (3).

The next theorem help us to find the optimal solution by a binary search on the set of the penalties.

Theorem 3. If $D_{r}$ is a feasible solution to the problem (3), then $D_{r+1}$ is also feasible.

Proof. On the contrary, suppose that $D_{r+1}$ is not feasible to (3). Hence $P^{0}$ is not efficient in $G=\left(N, A, D_{r+1}\right)$. Thus there exists a path $P$ from $s$ to $t$ such that $D_{r+1}(P) \leq D_{r+1}\left(P^{0}\right)$. Analysis similar to that in the proof of Theorem 2 shows that $D_{r}(P) \leq D_{r}\left(P^{0}\right)$ which contradicts the feasibility of $D_{r}$ for (3).

Based on the previous results, we propose an algorithm to solve the IMSPP under the BWHD. We find the minimum value of $r \in\{1, \ldots, q m\}$ such that $P^{0}$ is an efficient path in $G=\left(N, A, D_{r}\right)$. For checking this condition, we can use the proposed algorithm in [4] to find all efficient paths from $s$ to $t$ in $G=\left(N, A, D_{r}\right)$. According to Theorem 3, the minimum value of $r$ can be found by a binary 
search on the set of the penalties. We now state our proposed algorithm formally.

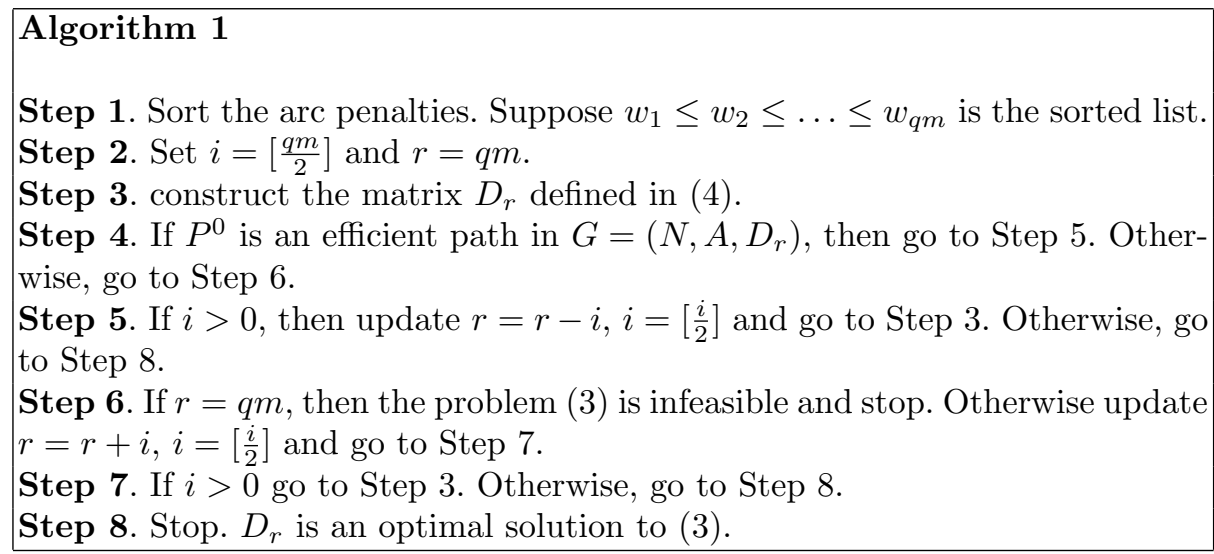

To analyze the complexity of the algorithm, note that the number of the iterations is $O(\log (q m))=O(\log (q n))$ and in each iteration an MSPP is solved. Hence if an MSPP can be solved in $T$ time, then the complexity of the algorithm is $O(T \log (q n))$.

Theorem 4. Algorithm 1 solves the IMSPP under the BWHD in $O(T \log (q n))$ time.

\section{Inverse multi-objective minimum spanning tree problem under the BWHD}

The algorithm proposed in the previous section can be used for the inverse of the others multi-objective combinatorial optimization problems under the BWHD. For instance, consider the inverse multi-objective minimum spanning tree problem (IMMSTP). Let $G=(V, E, C)$ be a graph with $|V|=n$ nodes, $|E|=m$ edges and the cost matrix $C \in \mathbb{Z}^{m \times q}$. Assume that $T^{0}$ be a given spanning tree of $G$. The IMMSTP under the BWHD can be written as follows:

$$
\begin{aligned}
\min & H_{w}(C, D), \\
\text { s.t. } & T^{0} \text { is an efficient spanning tree of } G=(V, E, D), \\
& -L^{k}(a) \leq D^{k}(a)-C^{k}(a) \leq U^{k}(a), \quad \forall a \in E, \quad \forall k \in\{1, \ldots, q\} \\
& D \in \mathbb{Z}^{m \times q} .
\end{aligned}
$$

For each $k \in\{1, \ldots, q\}$ and $r \in\{1, \ldots, q m\}$, the set $A_{r}^{k}$ is exactly the same as the previous section and The matrix $D_{r}$ is defined similar to (4) as follows:

$$
D_{r}^{k}(a)= \begin{cases}C^{k}(a) & \text { if } \quad a \in E \backslash A_{r}^{k}, \\ C^{k}(a)+U^{k}(a) & \text { if } a \in A_{r}^{k} \backslash T^{0}, \\ C^{k}(a)-L^{k}(a) & \text { if } \quad a \in A_{r}^{k} \cap T^{0} .\end{cases}
$$


Similarly, Theorem 2 ,Corollary 1 and Theorem 3 can be concluded for the problem (6). Consequently, Algorithm 1 can be applied for IMMSTP under the BWHD with this difference that in Step 4 we must investigate the efficiency of the spanning tree $T^{0}$ for $G=\left(V, E, D_{r}\right)$. It can be done by solving a multiobjective minimum spanning tree problem. We can use the Prim's spanning tree algorithm presented in [6].

\section{Conclusion}

In this article, the Inverse multi-objective shortest path problem under the bottleneck type weighted Hamming distance is considered. We proposed an algorithm based on the binary search technique to solve the inverse problem.

This work can be extended in different ways. For instance, other distances can be use. It is also possible to apply our proposed algorithm to solve the inverse of other problems.

\section{Acknowledgments}

The authors would like to thank the anonymous referees for their valuable comments to improve the paper.

\section{References}

1. R.K. Ahuja, J.B. Orlin, Inverse optimization, Operation Research 49, pp. 771-783, 2001.

2. D. Burton, $\mathrm{Ph}$. L. Toint, On an instance of the inverse shortest paths problem, Mathematical Programming 53, pp. 45-61, 1992.

3. C.W. Duin, A. Volgenant, Some inverse optimization problems under the Hamming distance, European Journal of Operational Research 170, pp. 887-899, 2006.

4. M. Ehrgott, Multicriteria optimization, Springer-Verlage, Berlin, Germany, 2005.

5. A. Farago, A. Szentesi, and B. Szviatovszki, Inverse optimization in high-speed networks, Discrete Applied Mathematics 129, pp. 83-98, 2003.

6. J. Prim, Shortest connection networks and some generalizations, Bell System Technics Journal 36, pp. 1389-1401.

7. J. Roland, Y. D. Smet, J. R. Figueira, Inverse multi-objective combinatorial optimization, Discrete Applied Mathematics 161, pp. 2764-2771, 2013.

8. P. Serafini, Some considerations about computational complexity for multi objective combinatorial problems, Recent advances and historical development of vector optimization, volume 294 of Lecture Notes in Economics and Mathematical Systems, pp. 222-232, 1986.

9. J. Tayyebi, M. Aman, On inverse linear programming problems under the bottleneck-type weighted Hamming distance, Discrete Applied Mathematics, DOI: 10.1016/j.dam.2015.12.017, 2015.

10. D.C. Wei, An optimized Floyd algorithm for the shortest path problem, Journal of Networks 5,pp. 1496-1504, 2010. 
11. S. Xu, J. Zhang, An Inverse Problem of the Weighted Shortest Path Problem, Japan Journal of Industrial Applied Mathematics 12, pp. 47-59, 1995.

12. J. Z. Zhang, Z. Ma, C. Yang, A column generation method for inverse shortest path problems, Zeitschrift fr Operations Research 41, pp. 347-358, 1995.

13. J. Z. Zhang, Z. Ma, A network flow method for solving some inverse combinatorial optimization problems, Optimization: A Journal of Mathematical Programming and Operations Research 37, pp. 59-72, 1996. 